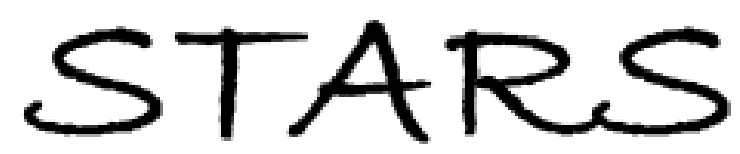

University of Central Florida

STARS

$1-1-2013$

\title{
Quantum cascade laser intracavity absorption spectrometer for trace gas sensing
}
A. V. Muraviev
University of Central Florida
D. E. Maukonen
University of Central Florida
C. J. Fredricksen
University of Central Florida
Gautam Medhi
University of Central Florida
R. E. Peale
University of Central Florida

Find similar works at: https://stars.library.ucf.edu/facultybib2010

University of Central Florida Libraries http://library.ucf.edu

This Article is brought to you for free and open access by the Faculty Bibliography at STARS. It has been accepted for inclusion in Faculty Bibliography 2010 s by an authorized administrator of STARS. For more information, please contactSTARS@ucf.edu.

\section{Recommended Citation}

Muraviev, A. V.; Maukonen, D. E.; Fredricksen, C. J.; Medhi, Gautam; and Peale, R. E., "Quantum cascade laser intracavity absorption spectrometer for trace gas sensing" (2013). Faculty Bibliography 2010 s. 4443. https://stars.library.ucf.edu/facultybib2010/4443

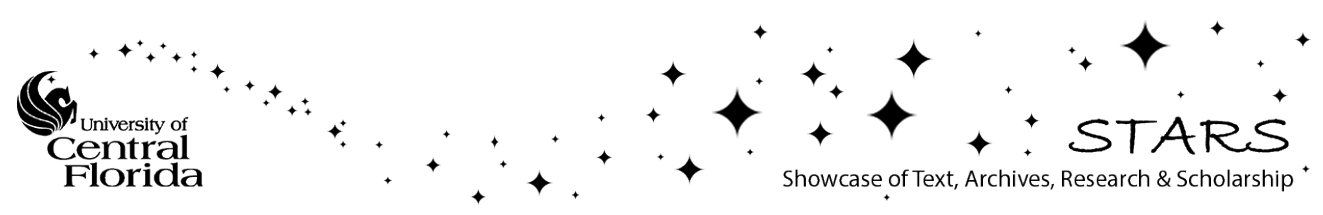




\section{Quantum cascade laser intracavity absorption spectrometer for trace gas sensing}

Cite as: Appl. Phys. Lett. 103, 09111 (2013); https://doi.org/10.1063/1.4819764

Submitted: 10 May 2013. Accepted: 16 August 2013 . Published Online: 29 August 2013

A. V. Muraviev, D. E. Maukonen, C. J. Fredricksen, Gautam Medhi, and R. E. Peale

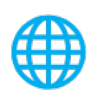

\section{ARTICLES YOU MAY BE INTERESTED IN}

Fast, precise, and widely tunable frequency control of an optical parametric oscillator referenced to a frequency comb

Review of Scientific Instruments 88, 033101 (2017); https://doi.org/10.1063/1.4977049

\section{Applied Physics Reviews} Now accepting original research 


\title{
Quantum cascade laser intracavity absorption spectrometer for trace gas sensing
}

\author{
A. V. Muraviev, D. E. Maukonen, C. J. Fredricksen, Gautam Medhi, and R. E. Peale \\ Department of Physics, University of Central Florida, Orlando, Florida 32816, USA
}

(Received 10 May 2013; accepted 16 August 2013; published online 29 August 2013)

\begin{abstract}
A mid-infrared intracavity laser absorption spectrometer for trace gas sensing is demonstrated. An external-cavity multi-mode quantum cascade laser with central wavelength $8.0 \mu \mathrm{m}$ was combined with a scanning Fabry-Perot interferometer, which analyzed the change of the laser emission spectrum caused by introducing an analyte inside the cavity. The detection mechanism is based on monitoring the laser spectrum dynamics at adiabatically changing laser conditions in long pulse operation mode. Fast acquisition and vapor exchange allow nearly real-time analyte detection. Sensitivity at the level of $1 \times 10^{-5} \mathrm{~cm}^{-1}$ was demonstrated based on a weak water vapor absorption line. (C) 2013 AIP Publishing LLC. [http://dx.doi.org/10.1063/1.4819764]
\end{abstract}

Intracavity Laser Absorption Spectroscopy (ICLAS) is well-established for detecting weak absorbers. ${ }^{1}$ A sample with sharp absorption lines is introduced into the open external cavity of a multimode laser. Gain compensates for cavity losses, enabling much longer effective path lengths than can be achieved using passive cavity approaches, such as ring-down spectroscopy. This enhances sensitivity, allowing detection at absorption coefficients as low as $10^{-10}-10^{-11} \mathrm{~cm}^{-1}$. 2,3 The highest optical path reported was $70000 \mathrm{~km}$, established with a CW dye laser. ${ }^{4}$

Since its conception, ${ }^{5}$ ICLAS has been demonstrated using gain media that include Ti:sapphire laser, ${ }^{2}$ dye lasers, ${ }^{6}$ color center lasers, ${ }^{7} \mathrm{Nd}^{3+}$ glass lasers, ${ }^{8}$ diode lasers, ${ }^{9}$ doped fiber lasers, ${ }^{10}$ and other solid state lasers. Previous demonstrations have been in the ultraviolet to near infrared wavelength range and in the THz. ${ }^{11}$ The mid-IR molecular fingerprint region has remained largely unexplored by this technique until recently. ${ }^{12-17}$ A mid-IR ICLAS system would have broad application in defense, security, environmental monitoring, medical diagnostics, etc.

Quantum Cascade Lasers (QCLs) ${ }^{18}$ are the most promising mid-IR laser sources for ICLAS due to their broadband emission spectra, wide tunability, high output power, high duty cycle, and room temperature operation. ${ }^{19}$ The molecular fingerprint region $(3-12 \mu \mathrm{m})$, where fundamental rotationalvibrational transitions of most molecules lie, is mostly accessible using QCLs, which provide opportunity for constructing portable hand held sensors. ${ }^{20}$ Working at the wavelengths corresponding to the vibrational fundamentals gives the highest possible absorption cross sections and improves the sensitivity over sensors based on detection of overtone bands at shorter wavelengths.

ICLAS is enabled by operating QCLs in an external cavity. The first external cavity QCL (EC-QCL) was demonstrated in 2001 at cryogenic temperature, ${ }^{21}$ and since then many groups ${ }^{22-26}$ and companies have implemented it. These works have emphasized use of a diffraction grating as external feedback reflector for fine tuning the output spectrum over a dense quasi-continuous longitudinal mode spectrum.

We demonstrate here a QCL-based ICLAS sensor using an open semiconfocal laser cavity, operating in long pulse mode. The spectral dynamics of the laser emission spectrum of the laser in the presence of an intracavity absorber is analyzed by a scanning Fabry-Perot (FP) interferometer. With this configuration, we estimate sensitivity to absorption coefficients as small as $1 \times 10^{-5} \mathrm{~cm}^{-1}$.

Fig. 1 presents a schematic of our experimental setup. The external cavity quantum cascade laser uses a gold-coated off-axis $90^{\circ}$ parabolic mirror to collimate the diverging beam from the active laser crystal. A plane gold mirror with a $1 \mathrm{~mm}$ diameter out-coupling hole drilled in its center completes the cavity (a high-reflection (HR) coated $\mathrm{ZnSe}$ also works as an output coupler, but the hole mirror was used in this demonstration). The active chip was purchased from Maxion Technologies, Inc. with high reflecting coating on the back facet and anti-reflection coating on the front facet. The use of the parabolic mirror and the absence of intracavity windows avoid unwanted reflections by dielectric interfaces, which cause an undesirably sparse spectrum. ${ }^{14}$

The strong temperature dependence of the QCL spectrum requires strict thermal stabilization to achieve the highest sensitivity. The QCL temperature rises rapidly during the first $1 \mathrm{~ms}$ of excitation and stabilizes after about $10 \mathrm{~ms}$, as indicated by the time-dependent mode spectrum. ${ }^{15}$ This temperature rise has been found to be very repeatable pulse to pulse if the average temperature of the laser mount is stable, which we achieve using a thermoelectric cooler with feedback from thermistors, whose values are monitored with 6 digit precision. The achieved temperature stability for the mount is $1 \mathrm{mK}$, which provided sufficient stability in spectrum and intensity to achieve high sensitivity detection.

The optical system is isolated inside a sealed enclosure. We used dry nitrogen to purge the enclosure and as a carrier for analyte vapor. Positive pressure in the enclosure helps confine the sample within a window-less intracavity gas cell with open ends that is placed inside the cavity. Sample vapor is confined to the gas cell by balancing the flow of sample and enclosure purge gas.

By diluting a certain volume of pure saturated analyte vapor in dry-nitrogen, we achieve the desired analyte concentration. An automated pump and valve system provides fast exchange between sample and reference vapors, which 


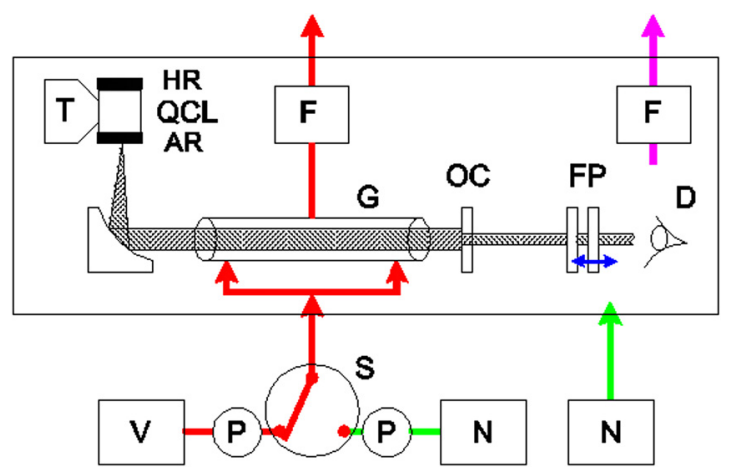

FIG. 1. Schematic of experimental setup. The optics are contained within a sealed enclosure with feedthroughs for electronics and gas lines. Labeled blocks are T: thermoelectric cooler; QCL: quantum cascade laser; HR: high-reflection coating; AR: anti-reflection coating; G: open-ended vapor cell; OC: output coupler; D: detector; F: flow meters; V: analyte vapor source disolved in dry nitrogen; N: dry nitrogen supplies; P: pumps; S: solenoid valve; FP: Fabry-Perot interferometer.

is synchronized with the data acquisition. The vapor exchange rate is $5-10 \mathrm{~s}$ for the $30 \mathrm{ml}$ gas-cell volume. Fast spectrum acquisition combined with fast vapor exchange allows nearly real time monitoring of the analyte-specific absorption. Averaging of spectral measurements for repeated synchronous vapor exchange improves the signal-to-noise ratio and enhances overall system sensitivity.

The QCL is driven by $\sim 10 \mathrm{~ms}$ pulses from a low-noise current supply. The voltage waveform applied to the QCL is monitored on an oscilloscope to indicate radiation feedback into the active region of the QCL chip, as has been independently reported in Ref. 17. The resolution and pulse-to-pulse stability of these voltage measurements is hundreds of $\mu \mathrm{V}$ at 600-1000 mA QCL current.

The laser emission spectrum is determined using a scanning Fabry-Perot interferometer ${ }^{13,14}$ and a $77 \mathrm{~K} \mathrm{HgCdTe} \mathrm{de-}$ tector at the output of the laser cavity. The intensity of the laser emission transmitted by the Fabry-Perot changes with time during the $10 \mathrm{~ms}$ laser pulse due to the aforementioned thermally induced spectral changes. For each step of the Fabry-Perot mirror, this entire waveform is recorded, acquiring the complete time dependence of the QCL spectrum during the pulse. The time resolution of the detector is $1 \mu \mathrm{s}$, but we have reduced the acquisition bandwidth to $25 \mathrm{kHz}$ to eliminate high frequency noise. The Fabry-Perot interferometer is scanned through its free spectral range, which is made to exceed the QCL gain bandwidth by selecting a sufficiently small resonance order for the given finesse. The spectral resolution achieved at this resonance order is $\sim 0.5 \mathrm{~cm}^{-1}$. We oversample the Fabry-Perot scan at an interval equivalent to a $0.18 \mathrm{~cm}^{-1}$ resolution. The acquisition time to scan over and collect the full laser emission band at this resolution was $\sim 5 \mathrm{~s}$.

The emission spectrum spans the wavenumber range $1240-1260 \mathrm{~cm}^{-1}$ and can be tuned slightly by changing the operating current and active chip cold-plate temperature. Typical dynamics of the system emission spectrum are presented in Fig. 2. The spectrum consists of up to $1 \mathrm{~ms}$ continuous fragments followed by jumps to new locations. These mode hops are due to the thermal change in the optical length of the laser active element during the pulse, which

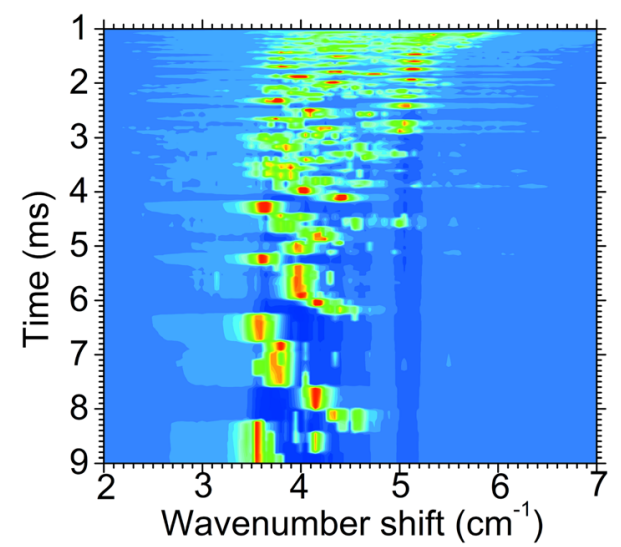

FIG. 2. Typical time dependence of the laser spectrum measured with FP interferometer. The laser pulse starts at $\mathrm{t}=1 \mathrm{~ms}$.

appears due to the remaining weak reflection at the AR-coated facet. Mode hopping and continuous drift together provide continuous coverage when integrated over the emission spectral range.

We used water vapor as the first analyte to demonstrate the system sensing capability. In the spectral range of our QCL, water vapor has weak absorption lines at 1242.8 and $1244.1 \mathrm{~cm}^{-1} .{ }^{27}$ We demonstrate two approaches to spectral data analysis after the exposure to this analyte.

The first approach is to monitor one of the continuous spectrum segments between mode hopping events in a spectral range matching the absorption lines. Fig. 3 presents a fragment of the emission spectrum $140 \mu \mathrm{s}$ long, taken in the time interval between 2.1 and $2.24 \mathrm{~ms}$ from the beginning of the laser pulse, where the spectrum is wide with a large number of individual modes. The laser current was $700 \mathrm{~mA}$ and the laser mount temperature $18 \mathrm{C}$. Fig. 3 (top) is the

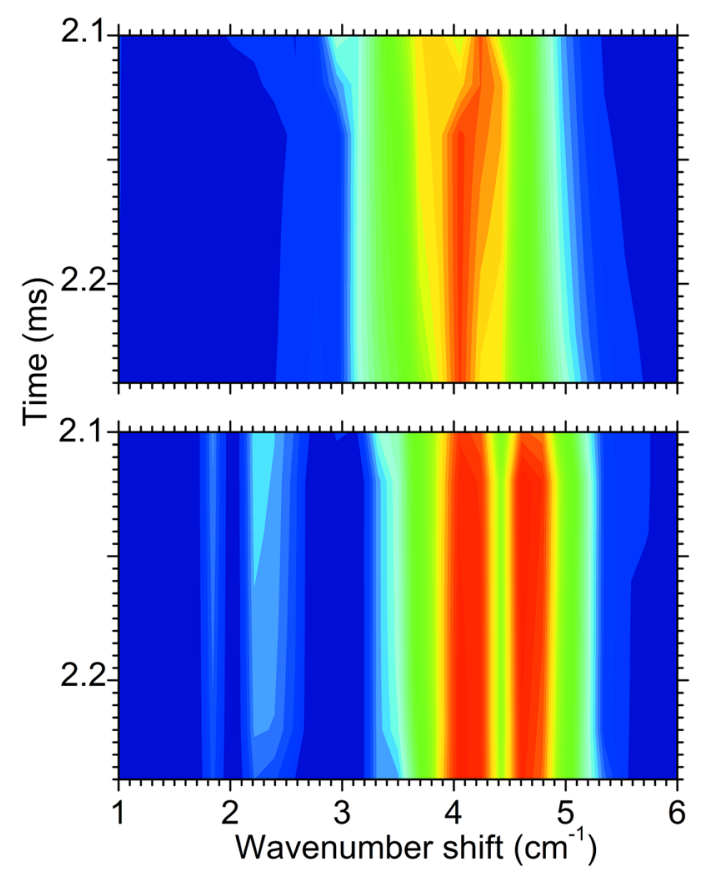

FIG. 3. (top) Fragment of laser spectrum with dry nitrogen inside the gas cell. (bottom) The same fragment with water vapor in the gas cell. The origin of the Fabry-Perot frequency scale was determined from subsequent calibration to be $1239.7 \mathrm{~cm}^{-1}$. 
reference emission spectrum taken for dry nitrogen in the cavity. Fig. 3 (bottom) is the emission spectrum when water vapor at 50\% humidity level is present inside the gas cell within the cavity. This last spectrum reveals the characteristic sharp absorption line of water vapor. Simultaneously, the presence of absorption redistributes the power over the modes by suppressing the gain of some of them. Suppressing of the strongest modes allows satellites to grow, broadening the emission spectrum.

Fig. 4(a) presents the time integral of Fig. 3 from 2.1 to $2.24 \mathrm{~ms}$ before and after water vapor is introduced to the cell. Fig. 4(c) is the reference water absorption spectrum. When water vapor is present, a dip in the laser spectrum clearly coincides with the water line at $1244.1 \mathrm{~cm}^{-1}$. The evident broadening of the laser spectrum when water vapor is in the cavity interestingly tends to avoid the position of the second, weaker water line at $1242.8 \mathrm{~cm}^{-1}$. This peak has an absorption coefficient of only $\sim 1 \times 10^{-5} \mathrm{~cm}^{-1}$, which we thus estimate to be the achieved system sensitivity (the frequency scale in Fig. 4 was calibrated by comparing the laser spectrum measured with the Fabry-Perot with that measured by a Fourier Transform Spectrometer for the same laser conditions. The $\sim 0.5 \mathrm{~cm}^{-1}$ accuracy of this approach is sufficient for unambiguous correlation with the strong line in the reference spectrum, which was then used to fine tune the calibration).

The second approach is based on the dynamic tuning of the laser spectrum through the intracavity absorption line near the end of the pulse. The differential spectrum and its derivative (Fig. 4(b)) are taken for the time segment 6.2-8.2 ms (see Fig. 2). Here, the spectrum consists of a single narrow line swept over a wide spectral range in the direction of ascending frequency. This approach differs from the first by measuring and integrating the effect of the absorber on the dynamics of the relatively narrow laser spectrum passing through the absorption line. The sweep of the spectrum

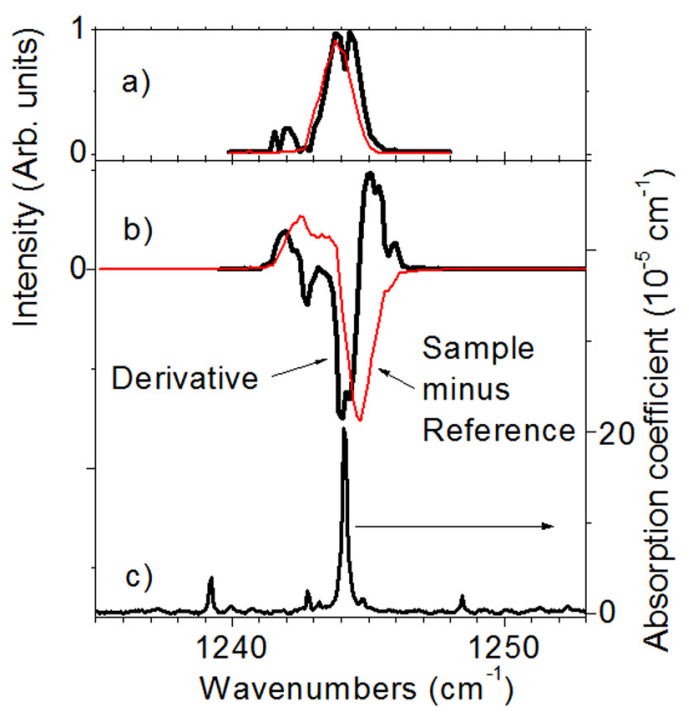

FIG. 4. (a) Reference (light curve) and sample (heavy curve) spectra integrated over the $140 \mu$ s duration of a wide laser spectrum taken near the beginning of the pulse $(\tau=2.1-2.24 \mathrm{~ms})$. The sample was $\mathrm{N}_{2}$ with $\sim 50 \%$ humidity and the reference was dry $\mathrm{N}_{2}$. (b) Difference spectrum and its derivative for the spectrum fragment integrated near the end of the pulse ( $\tau=6.2-8.2 \mathrm{~ms}$ ), where the laser spectrum is narrow but adiabatically swept over the wide range. (c) Reference water vapor spectrum. ${ }^{27}$ is observed to slow down and then accelerate as it approaches and then passes the absorption line. This increases the integrated emission spectral power density before the absorption line, and decreases it afterward, as shown by the time integral of the difference spectrum (Fig. 4(b)). The absorption line is more apparent in the derivative of this curve with respect to frequency (Fig. 4(b)). The peak of the derivative represents the fastest drop of the laser spectral power density as the spectrum sweeps across the analyte absorption line. It matches the position and width of the water vapor absorption line at $1244.1 \mathrm{~cm}^{-1}$ on the reference spectrum. ${ }^{27}$ The weak line at $1242.8 \mathrm{~cm}^{-1}$ is also clearly visible, which establishes that the achieved sensitivity to absorption coefficients is at the level of $\sim 10^{-5} \mathrm{~cm}^{-1}$, as before.

To smooth and broaden the spectrum for Fig. 4(b), data for laser drive currents of 680,700 , and $710 \mathrm{~mA}$ were collected and averaged. Each was collected for 5 consecutive vapor exchange cycles. The $700 \mathrm{~mA}$ spectrum was measured 3 times, giving a total of 25 vapor exchange cycles, or a signal to noise improvement of about 5 .

For reasonably strong cross sections of $10^{-17} \mathrm{~cm}^{2}$ (e.g., the strongest line of $\mathrm{CO}_{2}$ near 4.5 microns wavelength has cross section $1.4 \times 10^{-17} \mathrm{~cm}^{2}$ (Ref. 27)), our result implies sensitivity to concentrations of $10^{12} \mathrm{~cm}^{-3}$, or $40 \mathrm{ppb}$ mixing ratio compared with the atmosphere at standard conditions. For our $25 \mathrm{kHz}$ measurement bandwidth, we estimate a noise equivalent absorption coefficient of $\sim 2 \times 10^{-6} \mathrm{~cm}^{-1} / \mathrm{Hz}^{-1 / 2}$.

In summary, we have demonstrated an intracavity absorption spectrometer based on a quantum-cascade-laser operating in the long pulse regime, allowing the multi-mode spectrum to be dynamically tuned through the analyte absorption line due to active crystal heating. The sample handling and data acquisition scheme allow nearly real time monitoring. Two approaches to detection were compared, both indicating a sensitivity to sharp-line vapor spectra at an absorption coefficient level of $1 \times 10^{-5} \mathrm{~cm}^{-1}$. This value might be dramatically improved by bandwidth limiting and by further improving the laser stability.

This project was supported by Army Phase I and II SBIRs (Dr. Dwight Woolard, PM), by matching funds from the Florida High Technology Corridor (I-4) program, by a grant from the Florida Space Institute, and by a grant from the Florida Space Grant Consortium.

${ }^{1}$ V. M. Baev, T. Latz, and P. E. Toschek, Appl. Phys. B 69, 171-202 (1999).

${ }^{2}$ A. Kachanov, A. Charvat, and F. Stoeckel, J. Opt. Soc. Am. B 11, 2412-2421 (1994).

${ }^{3}$ A. Garnache, A. Kachanov, and F. Stoeckel, Opt. Lett. 24, 826-828 (1999).

${ }^{4}$ J. Sierks, T. Latz, V. M. Baev, and P. E. Toschek, "Spectral dynamics of multi-mode dye lasers and single atom absorption," in International Quantum Electronics Conference - OSA Technical Digest Series (Optical Society of America, Washington DC, 1996).

${ }^{5}$ L. N. Pakhomycheva, E. A. Sviridenkov, A. F. Suchkov, L. V. Titova, and S. S. Churilov, J. Exp. Theor. Phys. Lett. 12, $43-45$ (1970).

${ }^{6}$ T. W. Hänsch, A. L. Schawlow, and P. E. Toschek, IEEE J. Quantum Electron. 8, 802-804 (1972).

${ }^{7}$ V. M. Baev, H. Schröder, and P. E. Toschek, Opt. Commun. 36, 57-62 (1981). 
${ }^{8}$ D. Bykov, V. P. Lopasov, Yu. S. Makushkin, L. N. Sinitsa, O. N. Ulenikov, and V. E. Zuev, J. Mol. Spectrosc. 94, 1-27 (1982).

${ }^{9}$ V. M. Baev, J. Eschner, E. Paeth, R. Schuler, and P. E. Toschek, Appl. Phys. B 55, 463-477 (1992).

${ }^{10}$ R. Böhm, A. Stephani, V. M. Baev, and P. E. Toschek, Opt. Lett. 18, 1955-1957 (1993).

${ }^{11}$ E. W. Nelson, S. H. Withers, A. V. Muravjov, R. C. Strijbos, R. E. Peale, S. G. Pavlov, V. N. Shastin, and C. J. Fredricksen, IEEE J. Quantum Electron. 37, 1525-1530 (2001).

${ }^{12}$ G. Medhi, A. V. Muravjov, H. Saxena, J. W. Cleary, C. J. Fredricksen, R. E. Peale, and O. Edwards, "Infrared intracavity laser absorption spectrometer," Proc. SPIE 7680, 24 (2010).

${ }^{13}$ G. Medhi, A. V. Muraviev, H. Saxena, J. W. Cleary, C. J. Fredricksen, R. E. Peale, and O. Edwards, "Infrared intracavity laser absorption spectrometer," in Proceedings of International Symposium on Spectral Sensing Research, Springfield, Missouri, 21-24 June 2010.

${ }^{14}$ G. Medhi, A. V. Muravjov, H. Saxena, C. J. Fredricksen, T. Brusentsova, R. E. Peale, and O. Edwards, "Intracavity laser absorption spectroscopy using mid-IR quantum cascade laser," Proc. SPIE 8032, 12-19 (2011).

${ }^{15}$ G. Medhi, C. J. Fredricksen, R. E. Peale, A. V. Muravjov, and O. J. Edwards, "Sensitivity of long-wave infrared intracavity laser absorption vapor detector," Proc. SPIE 8236, 55 (2012).
${ }^{16}$ A. Muraviev, D. E. Maukonen, C. J. Fredricksen, G. Medhi, and R. E. Peale, "Quantum cascade laser intracavity absorption sensor," Proc. SPIE 8710, 27 (2013).

${ }^{17}$ M. C. Phillips and M. S. Taubman, Opt. Lett. 37, 2664 (2012).

${ }^{18}$ J. Faist, F. Capasso, D. L. Sivco, C. Sirtori, A. L. Hutchinson, and A. Y. Cho, Science 264, 553-556 (1994).

${ }^{19}$ Q. Wen and K. H. Michaelian, Opt. Lett. 33, 1875-1877 (2008).

${ }^{20}$ A. Kosterev, R. F. Curl, F. K. Tittel, M. Rochat, M. Beck, D. Hofstetter, and J. Faist, Appl. Phys. B: Lasers Opt. 75, 351-357 (2002).

${ }^{21}$ A. Karpf and G. N. Rao, Appl. Opt. 48, 408-413 (2009).

${ }^{22}$ G. P. Luo, C. Peng, H. Q. Le, and S. S. Pei, Appl. Phys. Lett. 78, 2834-2836 (2001).

${ }^{23}$ G. Totschnig, F. Winter, V. Pustogov, J. Faist, and A. Muller, Opt. Lett. 27, 1788-1790 (2002).

${ }^{24}$ G. P. Luo, C. Peng, H. Q. Lee, S. S. Pei, H. Lee, W. Y. Hwang, B. Ishaug, and J. Zheng, IEEE J. Quantum Electron. 38, 486-494 (2002).

${ }^{25}$ R. Maulini, M. Beck, J. Faist, and E. Gini, Appl. Phys. Lett. 84, 1659-1661(2004).

${ }^{26} \mathrm{~T}$. Tsai and G. Wysocki, "Fast wavelength tuning of external cavity quantum cascade lasers," in Conference on Lasers and Electro-Optics and Quantum Electronics and Laser Science (CLEO/QELS) (2009).

${ }^{27} \mathrm{See}$ http://nwir.pnl.gov for "Northwest-infrared: Vapor phase infrared spectral library." 